AGH DRILLING, OIL, GAS • Vol. 34 • No. $2 \cdot 2017$

http://dx.doi.org/10.7494/drill.2017.34.2.385

Piotr Krzeczek*, Paweł Karasek**, Paweł Wojnarowski*

\title{
ANALYSIS IMPACT FRACTURING FLUID ACIDITY ON STRUCTURAL STRENGTH OF CROSSLINKED GEL COMPLEX
}

\section{INTRODUCTION}

Hydraulic fracturing is a technique used to stimulate the productivity of a well. A hydraulic fracture is a superimposed structure that remains undisturbed outside the fracture. Thus the effective permeability of a reservoir remains unchanged by this process. The increased wellbore radius increases its productivity, because a large contact surface between the well and the reservoir is created [1].

Generally, a hydraulic fracturing treatment involves pumping a proppant-free viscous fluid, or pad, usually water with some fluid additives to generate high viscosity, into a well faster than the fluid can escape into the formation so that the pressure rises and the rock breaks, creating artificial fractures or enlarging existing fractures [1].

After fracturing the formation, a propping agent, such as sand is added to the fluid to form a slurry that is pumped into the newly formed fractures in the formation to prevent them from closing when the pumping pressure is released. The proppant transportability of a base fluid depends on the type of viscosifying additives added to water base [2].

\section{FRACTURING FLUIDS}

The selection of a proper fracturing fluid is all about choices. It begins with choosing the pad volume where one must consider what and how much pad is required to create

* AGH University of Science and Technology, Faculty of Drilling, Oil and Gas, Krakow, Poland

** United Oilfield Services Sp. z o.o., Warszawa, Poland 
the desired fracture geometry. This is followed by choosing how much viscosity the fluid needs to have to provide sufficient fracture width, a desired net pressure to either treat some desired height growth or prevent breaking out into some undesirable zone, provide carrying capability to transport proppant from the wellbore to the fracture tip, and control fluid loss. In case where a gel filter cake cannot form the fracturing fluid viscosity may be the main mechanism for fluid loss control [6].

Fracturing fluids must meet a number of requirements simultaneously. They must be stable at high temperatures, pumping rates and shear rates. These severe conditions can cause the fluid to degrade and prematurely settle out the proppant before the fracturing operations is complete. Most commercially used fluids are aqueous liquids that have been either gelled or foamed [1].

There are several types of fracturing fluids and a wide and confusing range of fluid additives. The types of fluid include: water based, oil based, energized fluids, multi-phase emulsions, acid fluids [6].

Chemical Additives [7]:

- Control viscosity:

- Increase viscosity $=>$ Crosslinker

- Adjust time to develop viscosity => Delay agent

- Reduce viscosity => Breaker

- Control pH to maintain viscosity $=>$ pH Buffer

- Prevent premature loss of viscosity $=>$ Biocide/Bactericide

- Maintain viscosity at elevated temperature $=>$ HT stabilizer

- Formation compatibility:

- Prevent permeability loss $=>$ Clay stabilizer

- Leave formation water wet $=>$ Surfactant

- Prevent fluid incompatibility $=>$ Non-emulsifier

- Surface requirements:

- HHP requirements $=>$ Friction reducer

- Fluid requirements $=>$ Fluid Loss Additive

Linear gel is composed of water, a clay control agent and gelling agent such as Guar, HPG or HEC. Because these gelling agents are susceptible to bacteria growth biocide is also added. Chemical breakers are also added to reduce damage to the proppant pack. The main advantage of a linear gel is its low cost and improved viscosity characteristics. Fluid loss is controlled by a filter cake which builds on the fracture face as the fluid loses fluid to the formation. The main disadvantage is, as waterfracs, the low viscosity which results in a narrow fracture width. The main disadvantage when compared to waterfrac is that because the returned water has residual breaker the water is not reusable [6]. 


\section{Thickeners and gelling agents}

A gelling agent is also addressed as a viscosifying agent. A gelling agent refers to a material capable of forming the fracturing fluid into a gel, thereby increasing its viscosity. [2]. Suitable gelling agents include guar gum, xanthan gum, welan gum, locust bean gum. A suitable depolimerized gum is a depolymerized guar gum. The guar gum can be functionalized of modified to result in hydroxyethyl guar, hydroxypropyl guar, and carboxymethyl guar. Examples of water-soluble cellulose ethers include methyl cellulose, carboxymethyl cellulose (CMC), HEC, and hydroxyethyl carboxymethyl cellulose [2].

\section{pH control additives}

Buffers are necessary to adjust and maintain the $\mathrm{pH}$. Buffering agents can be salts of a weak acid and weak base. Examples are carbonates, bicarbonates, and hydrogen phosphates. Weak acids such as formic acid, fumaric acid, and sulfamic acid also are recommended [1].

Buffers provide control the $\mathrm{pH}$ of fluids, crosslinking agents will be affected by $\mathrm{pH}$, most fluids will break quicker when $\mathrm{pH}$ is lower, temperature stability of fluids is higher at higher $\mathrm{pH}$ values [7].

\section{Crosslinkers}

Crosslinkers are used to increase the molecular weight of the polymer by cross-linking the polymer backbone into a 3D structure as shown in Figure 1. This increases the base viscosity of the linear gel from less than $50 \mathrm{cps}$ into the $100 \mathrm{~s}$ or $1000 \mathrm{~s}$ of cps range. For guar- and CMHEC-based gels, boron and several metals including titanium and zirconium are used as cross-linkers. In addition to these materials, iron, chromium, aluminum will cross-link guar but are not commonly used. Iron is a major contaminant for fracturing fluid and is one of the metals that must be carefully controlled to prevent premature cross-linking. Each cross-linker has a unique reaction requirement and behavior [6].

\section{Borate crosslinkers [7]):}

- Borate salts cross link guar fluids to form stable compounds up to $350^{\circ} \mathrm{F}$.

- When $\mathrm{pH}$ is greater than 8.0 the viscosity increases in seconds.

- Optimum pH is 9-12.

- Viscosity is controlled by adjusting polymer concentration or Crosslinker concentration.

- Temperature stability is achieved with delay and $\mathrm{pH}$ control.

- Crosslinked fluids thin with shear and heat.

- Borate fluids tend to re-heal when cooled or shear is removed. 
Borate-crosslinked fluid used for fracturing consist of polysaccharide polymers crosslinked with borate salts. These gels are difficult to investigate with analytical instruments, so model diol chemical systems and model sugars have been used to study the formation of borate esters and crosslinking reactions. Borate crosslinks are viewed as rapid exchange equilibria of monoborate ion on the cis-diols of galactomannan polymers (guar) [5].

Borate in the form of boric acid, slowly soluble salts of $\mathrm{Ca}$ and $\mathrm{Mg}$, and organic borate complexes is, by far, the most common cross linker in use today. Figure 1 shows diagrammatically how the borate complexes with guar. As the figure shows, the borate source forms a tetrahedral form of borate ion when the $\mathrm{pH}$ of the base fluid is above about 8.2. These borate ions complex with the hydroxyl functionality on the polymer causing a 3D network to be formed, which tremendously increases the molecular weight and viscosity. Once this mechanism is understood, several things become apparent [6].

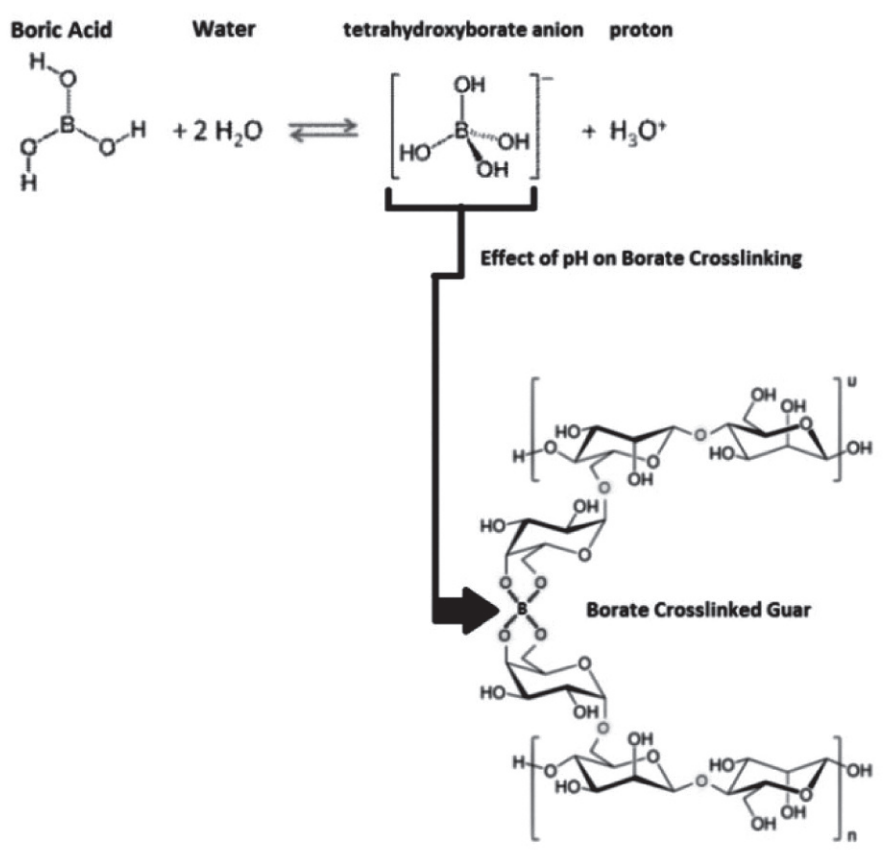

Fig. 1. Crosslinking process based on water, guar gum and borate [6] (modified)

The cross-linking is a function of $\mathrm{pH}$ that means it can be formed or reversed simply by adjusting the $\mathrm{pH}$. The optimum borate cross-linker efficiency is at a $\mathrm{pH}$ of about 10.5 . Because the cross-link is in equilibrium, it can be broken by shear in the tubing and will quickly build the cross-link again once the shear is dropped [6]. 


\section{Organometallic crosslinkers (Titanium and zirconium)}

Titanium and zirconium crosslinkers were originally developed for manufacturing explosive gels. The crosslinkers are manufactured in the form of metal ligand or chelant using various complexing agents. Both zirconium and tTitanium have coordination numbers of +4 so they form a strong covalent rigid bond with the polymers cis hydroxyls as shown in Figure 2. The various complexing agents allow the crosslinker to become active under a range of time, temperature and $\mathrm{pH}$ conditions. Titanium and Zirconium crosslinked fluids can be manufactured that are stable at $\mathrm{pH}$ levels from 3.5 to 10.5 and up to temperatures of $350^{\circ} \mathrm{F}$. The metallic crosslink is a strong covalent bond which makes the crosslink susceptible to high shear rates. Because it takes time for the metal to interact with the polymer the crosslink time can be delayed. The type of ligand used to complex the metal controls the delay time. Metallic crosslinked polymer systems can be built that cover a broad range of $\mathrm{pH}$ conditions. They are also much more stable at high temperatures. The molecular weight of the broken gel residue is much greater than that formed from linear or borate crosslinked gels. Any polymer which has hydroxyls in the cis position can be crosslinked with metallic crosslinkers [6].

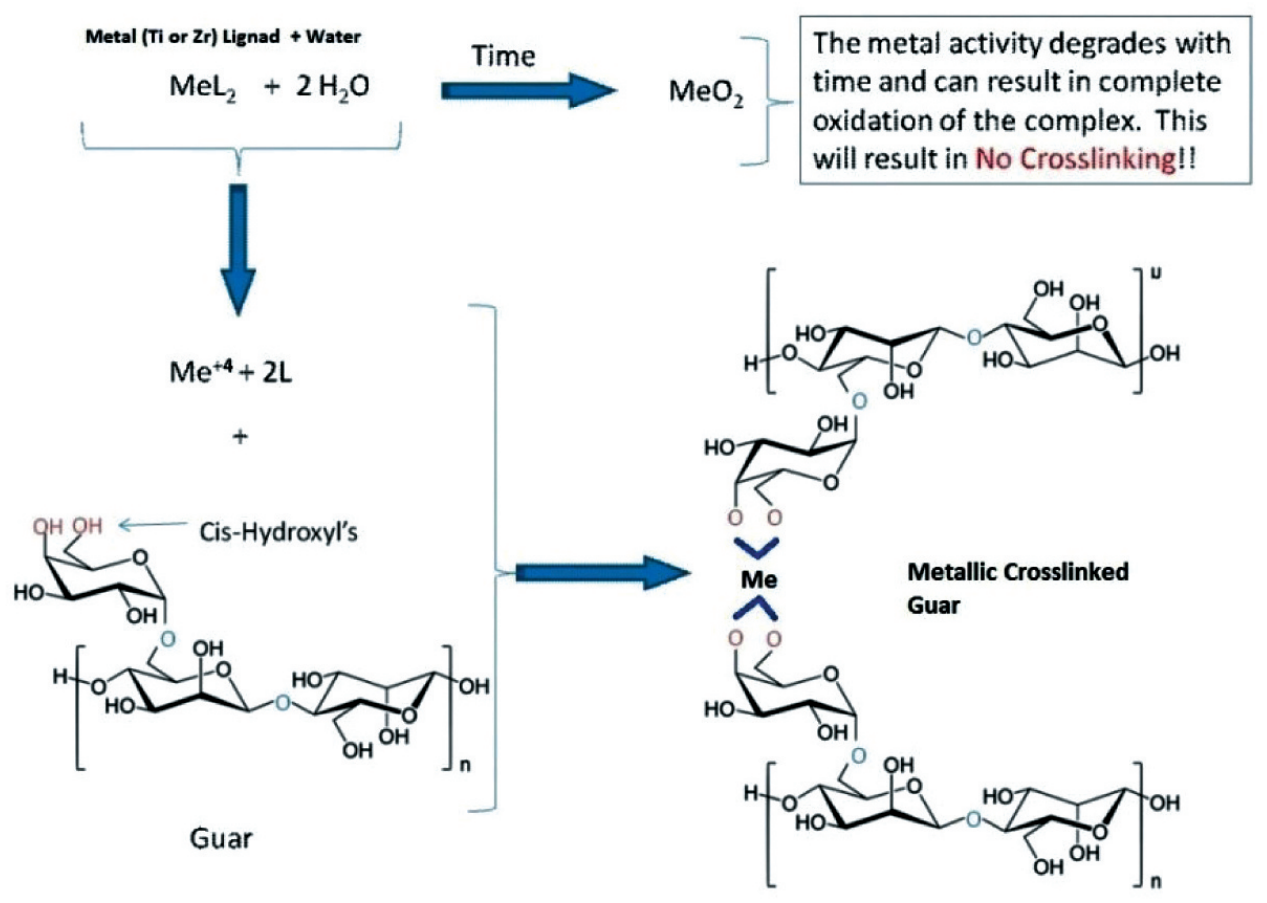

Fig. 2. Crosslinking mechanism for metallic crosslinkers onto guar [6] (modified) 
Factors affecting crosslink rate are: fluid temperature, $\mathrm{pH}$ (as it is shown in the Fig. 3), shear condition, concentration of reactants, crosslinker type, presence of competing ligands [7].

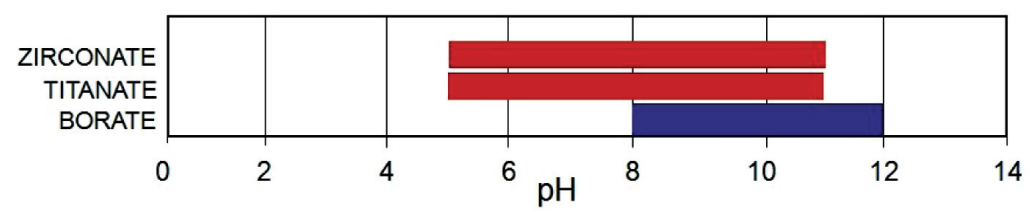

Fig. 3. Crosslinkers and theirs $\mathrm{pH}$ activity on crosslinking [7]

\section{LABORATORY EXPERIMENT}

\section{Description of laboratory equipment and the method used}

In order to measure basic properties of borate-crosslinked fluids a blender and $\mathrm{pH}$ measurement are needed. A pH meter equipped with an automated temperature controller was used to measure the $\mathrm{pH}$ of the fluid at surface condition. To prepare the fracture fluid in the laboratory we obviously required a laboratory scales and to apply a proper concentration of each component, two types of syringe: the bigger one was used for application of polymer and the smaller one for $\mathrm{pH}$ buffer and borate. The mixing time for every components was measured on the timer.

For the structure strength estimation, the hang-lip method was used. The delay time is defined as the time between the moment of adding the $\mathrm{pH}$ buffer until the fluid has reached sufficient viscosity to give a stable lip (Fig. 4). This method provides a zero shear rate evaluation of delay time. Borate fluids thin under shear so pumping viscosity would be lower than the hang lip viscosity [4].

Presented experiments are based on a 20 pounds of guar per 1000 gallons of fresh tap water. After 10 minutes mixing $250 \mathrm{ml}$ of fresh water added $1.31 \mathrm{~g}$ of polymer and for the next several samples in the same way, increasing amount of added buffer which was $\mathrm{NaOH}$. After thoroughly mixing additives with water, the $\mathrm{pH}$ of all fluid samples were measured. During mixing the linear gel with growing amount of the buffer, the $1 \mathrm{gpt}$ (gallon per thousand) crosslinker based on borate was added. In that case it was salt of $\mathrm{Na}$. The time the linear gel needs to transform into a cross-linked gel complex was measured from the start of mixing. Finally, the $\mathrm{pH}$ of fracturing fluid was measured once again. Every single measurement was conducted in the surface condition thus at the atmospheric pressure and at a temperature of approximately 20 Celsius. The pressure and temperature influence on fracturing fluid was not analyzed. Obviously those two parameters are enhanced during pumping. Due to rising temperature, the $\mathrm{pH}$ of fluid is increasing and in that case the crosslinking time will be increased as well. 


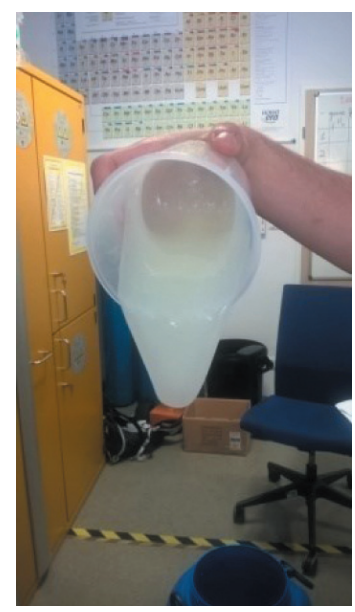

Fig. 4. Stable lip of crosslinked fracturing fluid (United Oilfield Services lab)

\section{RESULTS}

Table 1 shows data of added components, its quantity, measurement time and $\mathrm{pH}$ before and after the crosslinking process. Seven samples were prepared, each with growing concentration of buffer and with consequently rising $\mathrm{pH}$. For each sample of different $\mathrm{pH}$, borate crosslinker was added. Following that, the crosslinking time was measured and the final $\mathrm{pH}$ of the analyzed fluid was checked. Figure 5 shows influence of initial fluid $\mathrm{pH}$ on crosslinking time. The conducted research also gives information about the transition of $\mathrm{pH}$ between linear and crosslinked gel complex. It is visible in the Figure 6. In Figure 7 the influence of buffer quantity on the initial $\mathrm{pH}$ is also shown.

\section{Table 1}

Added components, its concentration and measurement of $\mathrm{pH}$ and crosslinking time for each sample of prepared fracturing fluid

\begin{tabular}{|c|c|c|c|c|c|c|c|c|c|}
\hline \multirow[t]{2}{*}{ No. } & \multirow{2}{*}{$\begin{array}{l}\text { Water } \\
{[\mathrm{ml}]}\end{array}$} & \multirow{2}{*}{$\begin{array}{l}\text { Guar } \\
\text { gum } \\
{[\mathrm{g}]}\end{array}$} & \multicolumn{2}{|c|}{$\begin{array}{c}\text { Buffer } \\
(\mathrm{NaOH})\end{array}$} & \multirow{2}{*}{$\begin{array}{c}\mathrm{pH} \text { (guar + water } \\
\text { + buffer) } \\
{[-]}\end{array}$} & \multicolumn{2}{|l|}{ Borate } & \multirow{2}{*}{$\begin{array}{c}\text { Crosslinking } \\
\text { time } \\
{[\mathrm{s}]}\end{array}$} & \multirow{2}{*}{$\begin{array}{l}\mathrm{pH} \\
{[-]}\end{array}$} \\
\hline & & & [gpt] & {$[\mathrm{ml}]$} & & {$[\mathrm{ml} / 250 \mathrm{ml}]$} & [gpt] & & \\
\hline 1 & 250 & 1.31 & 0 & 0 & 6.9 & 0.25 & 1 & 24 & 8.5 \\
\hline 2 & 250 & 1.31 & 1 & 0.25 & 8.7 & 0.25 & 1 & 35 & 9.1 \\
\hline 3 & 250 & 1.31 & 2 & 0.5 & 9.2 & 0.25 & 1 & 71 & 9.7 \\
\hline 4 & 250 & 1.31 & 3 & 0.75 & 9.7 & 0.25 & 1 & 118 & 10 \\
\hline 5 & 250 & 1.31 & 4 & 1 & 10.2 & 0.25 & 1 & 840 & 10.4 \\
\hline 6 & 250 & 1.31 & 6 & 1.5 & 10.6 & 0.25 & 1 & 774 & 10.8 \\
\hline 7 & 250 & 1.31 & 10 & 2.5 & 11.3 & 0.25 & 1 & - & - \\
\hline
\end{tabular}




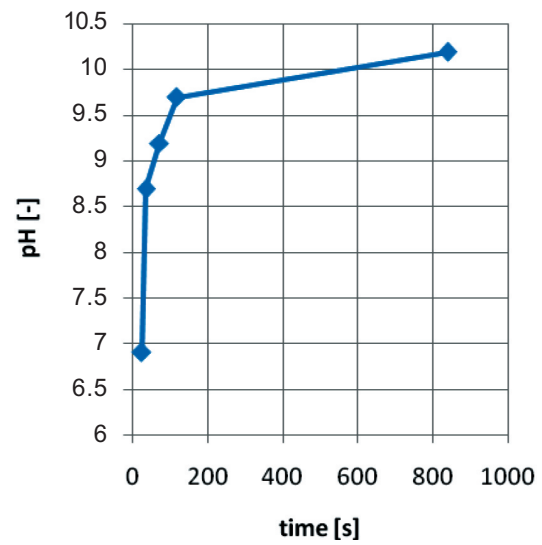

Fig. 5. Impact of the initial $\mathrm{pH}$ on the crosslinking time

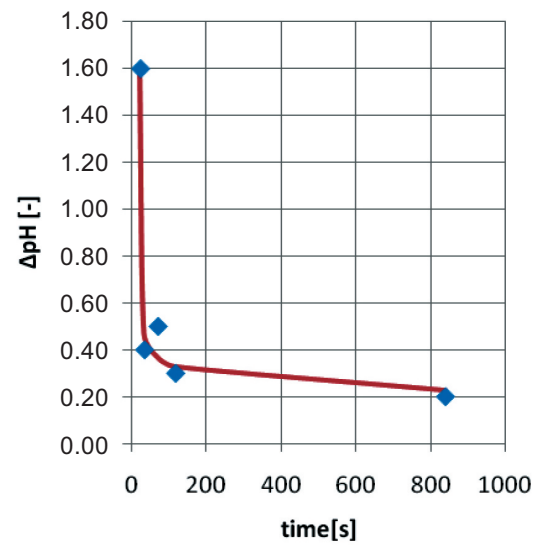

Fig. 6. Impact of transition $\mathrm{pH}$ on the crosslinking time

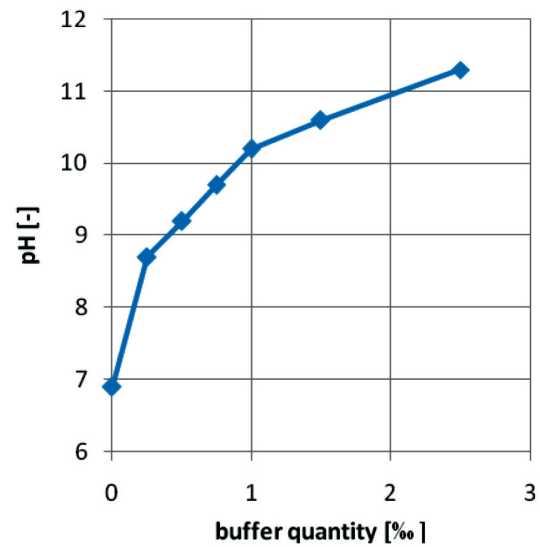

Fig. 7. Impact of buffer $(\mathrm{NaOH})$ quantity on the initial $\mathrm{pH}$ of fluid 
Table 2 shows measurement time and $\mathrm{pH}$ for different components of fracturing fluid. In this experiment 4 samples with a constant buffer concentration (which implies also constant initial $\mathrm{pH}$ ) were prepared. For these four samples of linear gel, rising quantity of borate crosslinker was added and crosslinking time and final $\mathrm{pH}$ of fluid were measured.

Table 2

Added components, its concentration and measurement of $\mathrm{pH}$ and crosslinking time for each sample of prepared fracturing fluid

\begin{tabular}{|c|c|c|c|c|c|c|c|c|c|}
\hline \multirow{2}{*}{ No. } & \multirow{2}{*}{$\begin{array}{c}\text { Water } \\
{[\mathrm{ml}]}\end{array}$} & \multirow{2}{*}{$\begin{array}{c}\text { Guar } \\
\text { gum } \\
{[\mathrm{g}]}\end{array}$} & \multicolumn{2}{|c|}{ Buffer $(\mathrm{NaOH})$} & \multirow{2}{*}{$\begin{array}{c}\mathrm{pH}(\text { guar }+ \\
\text { water } \\
+ \text { buffer }) \\
{[-]}\end{array}$} & \multicolumn{2}{|c|}{ Borate } & \multirow{2}{*}{$\begin{array}{c}\text { Crosslinking } \\
\text { time } \\
{[\mathrm{s}]}\end{array}$} & \multirow{2}{*}{$\begin{array}{l}\mathrm{pH} \\
{[-]}\end{array}$} \\
\hline & & & [gpt] & {$[\mathrm{ml}]$} & & {$[\mathrm{ml} / 250 \mathrm{ml}]$} & [gpt] & & \\
\hline 1 & 250 & 1.31 & 3 & 0.75 & 9.5 & 0.125 & 0.5 & 350 & 9.5 \\
\hline 2 & 250 & 1.31 & 3 & 0.75 & 9.5 & 0.375 & 1.5 & 52 & 9.7 \\
\hline 3 & 250 & 1.31 & 3 & 0.75 & 9.5 & 0.5 & 2 & 45 & 9.9 \\
\hline 4 & 250 & 1.31 & 3 & 0.75 & 9.5 & 0.875 & 3.5 & 23 & 10.1 \\
\hline
\end{tabular}

Figure 8 shows the influence of borate crosslinker quantity on crosslinking time. Furthermore, the conducted experiment gives also information about the acidity of borate crosslinker and it is visible in the Figure 9 as the transition of $\mathrm{pH}$.

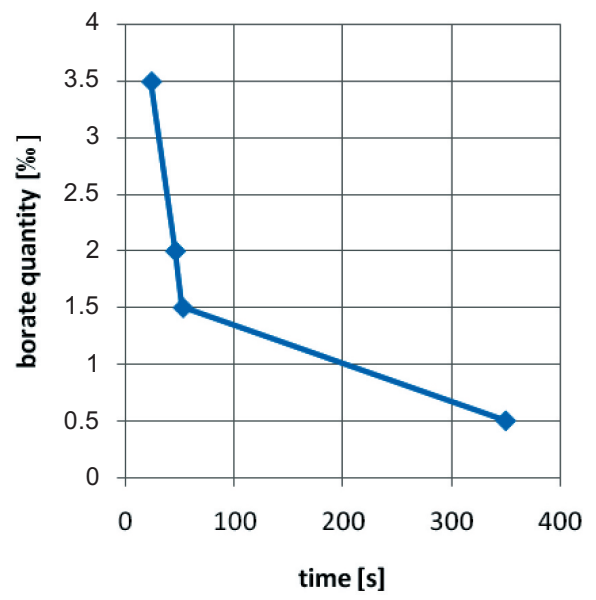

Fig. 8. Impact of borate quantity on the crosslinking time 


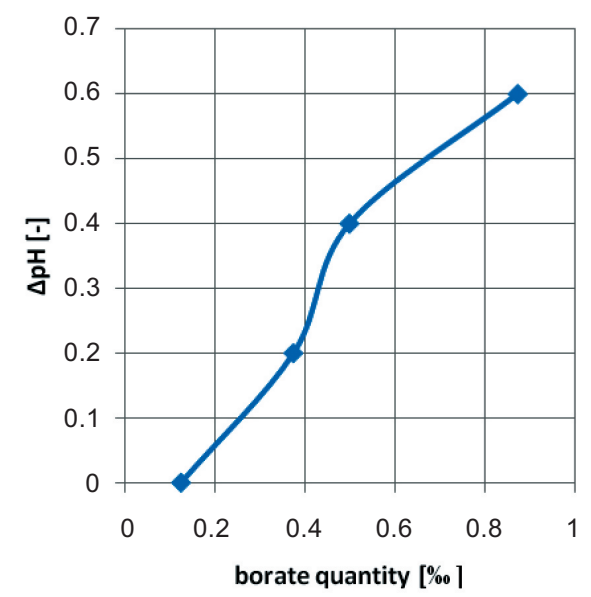

Fig. 9. Impact of borate quantity on the transition $\mathrm{pH}$ (between linear and crosslinked gel)

\section{SUMMARY}

In order to crack the rock and place the proppant in the conventional reservoir fracturing treatment, using the crosslinking gel complex is recommended. From engineering point of view, it is important to choose proper timing after which increased viscosity of fracturing fluid can occur, which is determined by crosslinking process. Generally, flow resistance of crosslinked gel is higher than linear gel. So that, it is recommendable first to cross perforation by linear fracturing fluid and then obtain fully crosslinking gel complex in order to let proppant deep into the made fracture.

Laboratory experiments presented in this work allow to evaluate the fracturing fluid $\mathrm{pH}$ impact on crosslinked gel complex creation. The initial fluid $\mathrm{pH}$ has influenced the crosslinking process. This is non-linear dependence. Linear gel $\mathrm{pH}$ grew from 7 to 11 . Crosslinking time of water-guar gum based gel has also increased. In case when $\mathrm{pH}$ reaches level of 11 there is high probability that crosslinking process might even exist in surface conditions. The lower linear gel $\mathrm{pH}$ caused faster reaction. As a result, higher crosslinker concentration gives faster crosslinking process and, what is more, $\mathrm{pH}$ of crosslinked gel is slightly increasing, which proves alkaline properties of borate crosslinker.

\section{Acknowledgments}

The research was conducted during the practice in the specialist laboratory thanks to United Oilfield Services. Authors wish to thank the management of United Oilfield Services for the permission to publish this research. 


\section{REFERENCES}

[1] Fink J.: Hydraulic Fracturing Chemicals and Fluid Technology. Elsevier Inc., 2013.

[2] Fink J.: Petroleum Engineer's Guide to Oil Field Chemicals and Fluids. Elsevier Inc., 2015, 2012.

[3] Fink J.: Water-Based Chemicals and Technology for Drilling, Completion, and Workover Fluids. Elsevier Inc., 2015.

[4] Nimerick K.H., Temple H.L., Card R.J.: New pH-Buffered Low Polymer Borate Crosslinked Fluids for Hydraulic Fracturing. SPE Journal, vol. 2, June 1997.

[5] Harris Ph.C. (Halliburton Services): Chemistry and Rheology of Borate-Crosslinked Fluids at Temperatures to 300F. SPE Journal of Petroleum Technology, vol. 45, March 1993.

[6] Smith M., Montgomery C.: Fracturing Fluids. Taylor \& Francis Group, LLC., 2013.

[7] United Oilfield Services notes, 2016. 